\title{
On Pointwise Approximation of Conjugate Functions by Some Hump Matrix Means of Conjugate Fourier Series
}

\author{
W. Lenski and B. Szal \\ Faculty of Mathematics, Computer Science and Econometrics, University of Zielona Góra, Ulica Szafrana 4a, \\ 65-516 Zielona Góra, Poland \\ Correspondence should be addressed to B. Szal; b.szal@wmie.uz.zgora.pl
}

Received 20 October 2014; Revised 7 January 2015; Accepted 21 January 2015

Academic Editor: Rodolfo H. Torres

Copyright (C) 2015 W. Łenski and B. Szal. This is an open access article distributed under the Creative Commons Attribution License, which permits unrestricted use, distribution, and reproduction in any medium, provided the original work is properly cited.

The results generalizing some theorems on $\left(N, p_{n}\right)(E, \gamma)$ summability are shown. The same degrees of pointwise approximation as in earlier papers by weaker assumptions on considered functions and examined summability methods are obtained. From presented pointwise results, the estimation on norm approximation is derived. Some special cases as corollaries are also formulated.

\section{Introduction}

Let $L^{p} \quad(1 \leq p<\infty)\left(\right.$ resp., $\left.L^{\infty}\right)$ be the class of all $2 \pi$-periodic real-valued functions integrable in the Lebesgue sense with $p$ th power (essentially bounded) over $Q=[-\pi, \pi]$ with the norm

$$
\begin{aligned}
\|f\| & :=\|f(\cdot)\|_{L^{p}} \\
& = \begin{cases}\left(\int_{Q}|f(t)|^{p} d t\right)^{1 / p} & \text { when } 1 \leq p<\infty, \\
\operatorname{ess} \sup _{t \in Q}|f(t)| & \text { when } p=\infty\end{cases}
\end{aligned}
$$

and consider the conjugate trigonometric Fourier series

$$
\widetilde{S} f(x):=\sum_{\nu=1}^{\infty}\left(a_{\nu}(f) \sin v x-b_{\nu}(f) \cos \nu x\right)
$$

with the partial sums $\widetilde{S}_{k} f$. We know that if $f \in L^{1}$ then

$$
\tilde{f}(x):=-\frac{1}{\pi} \int_{0}^{\pi} \psi_{x}(t) \frac{1}{2} \cot \frac{t}{2} d t=\lim _{\epsilon \rightarrow 0^{+}} \tilde{f}(x, \epsilon),
$$

where

$$
\tilde{f}(x, \epsilon):=-\frac{1}{\pi} \int_{\epsilon}^{\pi} \psi_{x}(t) \frac{1}{2} \cot \frac{t}{2} d t
$$

with

$$
\psi_{x}(t):=f(x+t)-f(x-t),
$$

exists for almost all $x$ [1, Th.(3.1)IV]

Let $A:=\left(a_{n, k}\right)$ and $B:=\left(b_{n, k}\right)$ be infinite lower triangular matrices of real numbers such that

$$
\begin{aligned}
& a_{n, k} \geq 0, \\
& b_{n, k} \geq 0,
\end{aligned}
$$

when $k=0,1,2, \ldots, n$,

$$
\begin{aligned}
& a_{n, k}=0, \\
& b_{n, k}=0,
\end{aligned}
$$

when $k>n$,

$$
\begin{aligned}
\sum_{k=0}^{n} a_{n, k} & =1, \\
\sum_{k=0}^{n} b_{n, k} & =1,
\end{aligned}
$$

where $n=0,1,2, \ldots$, 
and let, for $m=0,1,2, \ldots, n$,

$$
\begin{aligned}
& A_{n, m}=\sum_{k=0}^{m} a_{n, k}, \\
& \bar{A}_{n, m}=\sum_{k=m}^{n} a_{n, k} .
\end{aligned}
$$

Let the $A B$-transformation of $\widetilde{S}_{k} f$ be given by

$$
\widetilde{T}_{n, A, B} f(x):=\sum_{r=0}^{n} \sum_{k=0}^{r} a_{n, r} b_{r, k} \widetilde{S}_{k} f(x) \quad(n=0,1,2, \ldots) .
$$

We define two classes of sequences (see [2]).

Sequence $c:=\left(c_{k}\right)$ of nonnegative numbers tending to zero is called the Rest Bounded Variation Sequence, or briefly $c \in \mathrm{RBVS}$, if it has the property

$$
\sum_{k=m}^{\infty}\left|c_{k}-c_{k+1}\right| \leq K(c) c_{m}
$$

for all positive integer $m$, where $K(c)$ is a constant depending only on $c$.

Sequence $c:=\left(c_{k}\right)$ of nonnegative numbers will be called the Head Bounded Variation Sequence, or briefly $c \in$ HBVS, if it has the property

$$
\sum_{k=0}^{m-1}\left|c_{k}-c_{k+1}\right| \leq K(c) c_{m}
$$

for all positive integer $m$, or only for all $m \leq n$ if the sequence $c$ has only finite nonzero terms and the last nonzero term is $c_{n}$.

Now, we define the other classes of sequences.

Following Leindler [3], sequence $c:=\left(c_{r}\right)$ of nonnegative numbers tending to zero is called the Mean Rest Bounded Variation Sequence, or briefly $c \in$ MRBVS, if it has the property

$$
\sum_{r=m}^{\infty}\left|c_{r}-c_{r+1}\right| \leq K(c) \frac{1}{m+1} \sum_{r \geq m / 2}^{m} c_{r}
$$

for all positive integer $m$.

Analogously as in [4], sequence $c:=\left(c_{r}\right)$ of nonnegative numbers will be called the Mean Head Bounded Variation Sequence, or briefly $c \in \mathrm{MHBVS}$, if it has the property

$$
\sum_{r=0}^{n-m-1}\left|c_{r}-c_{r+1}\right| \leq K(c) \frac{1}{m+1} \sum_{r=n-m}^{n} c_{r}
$$

for all positive integers $m<n$, where the sequence $c$ has only finite nonzero terms and the last nonzero term is $c_{n}$. It is clear that (see [5])

RBVS $\subsetneq$ MRBVS,

HBVS $\varsubsetneqq$ MHBVS.
Consequently, we assume that the sequence $\left(K\left(\alpha_{n}\right)\right)_{n=0}^{\infty}$ is bounded, that is, that there exists a constant $K$ such that

$$
0 \leq K\left(\alpha_{n}\right) \leq K
$$

holds for all $n$, where $K\left(\alpha_{n}\right)$ denote the constants for the sequences $\alpha_{n}=\left(a_{n, r}\right)_{r=0}^{n}, n=0,1,2, \ldots$ appearing in the inequalities (11) and (12) as $K(c)$.

Now we can give the conditions to be used later on. We assume that for all $n$ and $0 \leq m<n$

$$
\begin{gathered}
\sum_{r=m}^{n-1}\left|a_{n, r}-a_{n, r+1}\right| \leq K \frac{1}{m+1} \sum_{r \geq m / 2}^{m} a_{n, r}, \\
\sum_{r=0}^{n-m-1}\left|a_{n, r}-a_{n, r+1}\right| \leq K \frac{1}{m+1} \sum_{r=n-m}^{n} a_{n, r}
\end{gathered}
$$

hold if $\left(a_{n, r}\right)_{r=0}^{n}$ belongs to MRBVS and MHBVS, for $n=$ $1,2, \ldots$, respectively.

We also define two hump matrices in the following way: a lower triangular matrix $C=\left(c_{n, k}\right)$ is called a maximal hump matrix if, for each $n$, there exists integer $k_{0}=k_{0}(n)$, such that $\left(c_{n, k}\right)_{k=0}^{k_{0}-1}$ is nondecreasing for $0 \leq k<k_{0}$ and $\left(c_{n, k}\right)_{k=k_{0}}^{n}$ is nonincreasing for $k_{0} \leq k \leq n$, but otherwise we will have a minimal hump matrix. The hump matrices were defined and considered in $[6,7]$.

As a measure of approximation of $\widetilde{f}$ by $\widetilde{T}_{n, A, B} f$, we use the pointwise modulus of continuity of $f$ in the space $L^{p}$ defined by the formula

$$
\begin{array}{ll}
\widetilde{w}_{x}^{p} f(\delta)_{\beta} & \begin{cases}\left\{\frac{1}{\delta} \int_{0}^{\delta}\left|\psi_{x}(u) \sin ^{\beta} \frac{u}{2}\right|^{p} d u\right\}^{1 / p} & \text { when } 1 \leq p<\infty, \\
=\operatorname{ess} \sup _{0<u \leq \delta}\left|\psi_{x}(u) \sin ^{\beta} \frac{u}{2}\right| & \text { when } p=\infty\end{cases}
\end{array}
$$

and the classical one

$$
\widetilde{\omega}_{\beta} f(\delta)_{L^{p}}=\sup _{0<t \leq \delta}\left\|\sin ^{\beta} \frac{t}{2} \psi \cdot(t)\right\|_{L^{p}} .
$$

The deviation $\widetilde{T}_{n, A} f-\tilde{f}=\widetilde{T}_{n, A, B} f-\tilde{f}$, with $b_{r, r}=1$ and 0 otherwise, was estimated at the point as well as in the norm of $L^{p}$ by Qureshi [8] and Lal and Nigam [9]. These results were generalized by Qureshi [10]. The next generalization was obtained by Lal [11]. In the case

$$
\begin{aligned}
& a_{n, r}=\frac{1}{n+1}, \quad \text { when } r=0,1,2, \ldots, n, \\
& a_{n, r}=0, \quad \text { when } r>n, \\
& b_{r, k}=\frac{\left(\begin{array}{l}
r \\
k
\end{array}\right) \gamma^{r}}{(1+\gamma)^{r}}, \quad \text { when } k=0,1,2, \ldots, r, \\
& b_{n, r}=0, \quad \text { when } k>r \text { with } \gamma>0,
\end{aligned}
$$

the deviation $\widetilde{T}_{n, A, B} f-\tilde{f}$ was estimated by Sonker and Singh [12] as follows. 
Theorem 1. Let $f(x)$ be a $2 \pi$-periodic, Lebesgue integrable function which belongs to the $\operatorname{Lip}(\alpha, p)$-class with $p \geq 1$ and $\alpha p \geq 1$. Then the degree of approximation of $\widetilde{f}(x)$, the conjugate of $f(x)$ by $(C, 1)(E, \gamma)$ means of series (2), is given by

$$
\begin{aligned}
& \left\|\frac{1}{n+1} \sum_{r=0}^{n} \frac{1}{(1+\gamma)^{r}} \sum_{k=0}^{r}\left(\begin{array}{l}
r \\
k
\end{array}\right) \gamma^{k} \widetilde{S}_{k} f(\cdot)-\tilde{f}(\cdot)\right\|_{L^{p}} \\
& =O\left((n+1)^{-\alpha+1 / p}\right),
\end{aligned}
$$

provided

$$
\begin{gathered}
\left\{\int_{0}^{\pi /(n+1)}\left(\frac{\left|\psi_{x}(t)\right|}{t^{\alpha}}\right)^{p} d t\right\}^{1 / p}=O\left((n+1)^{-1}\right), \\
\left\{\int_{\pi /(n+1)}^{\pi}\left(\frac{t^{-\delta}\left|\psi_{x}(t)\right|}{t^{\alpha}}\right)^{p} d t\right\}^{1 / p}=O\left((n+1)^{\delta}\right),
\end{gathered}
$$

where $\delta$ is an arbitrary positive number with $(\alpha+\delta) q+1<0$ and $p^{-1}+q^{-1}=1, p>1$.

In this paper we will consider the deviations $\widetilde{T}_{n, A, B} f(\cdot)-$ $\widetilde{f}(\cdot)$ and $\widetilde{T}_{n, A, B} f(\cdot)-\tilde{f}(\cdot, \pi /(n+1))$ in general form. In the theorems we formulate the general conditions for the functions and the modulus of continuity obtaining the same degrees of approximation as above and sometimes essentially better one. Finally, we also give some results on norm approximation with essentially better degrees of approximation. The obtained results generalize the results from $[4,9]$.

We will write $I_{1} \ll I_{2}$ if there exists positive constant $K$, sometimes depending on some parameters, such that $I_{1} \leq$ $\mathrm{KI}_{2}$.

\section{Statement of the Results}

Let

$$
L^{p}\left(\widetilde{w}_{x}\right)_{\beta}=\left\{f \in L^{p}: \widetilde{w}_{x}^{p} f(\delta)_{\beta} \leq \widetilde{w}_{x}(\delta)\right\},
$$

where $\widetilde{w}_{x}$ is a positive, with $\widetilde{w}_{x}(0)=0$, and nondecreasing continuous function.

We can now formulate our main results. At the beginning, we formulate the results on the degrees of pointwise summability of conjugate series.

Theorem 2. Let $f \in L^{1}$. If matrix $A$ is a maximal or minimal hump matrix with $k_{0}^{-1}=O\left(n^{-1}\right)$ such that $\left(a_{n, k}\right)_{k=0}^{n} \in$ MHBVS $\cup$ MRBVS and

$$
\left|\sum_{r=\mu}^{v} \sum_{k=0}^{r} b_{r, k} \cos \frac{(2 k+1) t}{2}\right| \ll \tau
$$

for $0 \leq \mu \leq \nu$, then

$$
\begin{aligned}
& \left|\widetilde{T}_{n, A, B} f(x)-\tilde{f}\left(x, \frac{\pi}{n+1}\right)\right| \\
& \quad=O\left((n+1)^{\beta}\left[\frac{1}{n+1} \sum_{k=0}^{n} \widetilde{w}_{x}^{1} f\left(\frac{\pi}{k+1}\right)_{\beta}\right]\right),
\end{aligned}
$$

for almost all considered $x$, where $\beta \in[0,1]$.
Theorem 3. Let $f \in L^{p}\left(\widetilde{w}_{x}\right)_{\beta}$ with $1<p<\infty$, and let $\widetilde{w}_{x}$ satisfy

$$
\begin{aligned}
& \left\{\int_{0}^{\pi /(n+1)}\left(\frac{\widetilde{w}_{x}(t)}{t \sin ^{\beta} t / 2}\right)^{p /(p-1)} d t\right\}^{(p-1) / p} \\
& =O\left((n+1)^{\beta+1 / p} \widetilde{w}_{x}\left(\frac{\pi}{n+1}\right)\right) \\
& \left\{\int_{0}^{\pi /(n+1)}\left(\frac{\left|\psi_{x}(t)\right|}{\widetilde{w}_{x}(t)}\right)^{p} \sin ^{\beta p} \frac{t}{2} d t\right\}^{1 / p} \\
& =O\left((n+1)^{-1 / p}\right)
\end{aligned}
$$

with some $\beta \geq 0$. If the entries of matrix $B$ satisfy condition (22) for $0 \leq \mu \leq \nu$ and if matrix $A$ is a maximal or minimal hump matrix with $k_{0}^{-1}=O\left(n^{-1}\right)$ such that $\left(a_{n, k}\right)_{k=0}^{n} \in$ MHBVS $\cup$ MRBVS, then

$$
\begin{aligned}
& \left|\widetilde{T}_{n, A, B} f(x)-\tilde{f}(x)\right| \\
& \quad=O\left((n+1)^{\beta}\left[\frac{1}{n+1} \sum_{k=0}^{n} \widetilde{w}_{x}\left(\frac{\pi}{k+1}\right)\right]\right),
\end{aligned}
$$

for almost all considered $x$ such that $\tilde{f}(x)$ exists.

Next, we formulate the results on estimates of $L^{p}$ norm of the deviation considered above. In case of the deviation $\widetilde{T}_{n, A, B} f(\cdot)-\widetilde{f}(\cdot)$, let

$$
L^{p}(\widetilde{\omega})_{\beta}=\left\{f \in L^{p}: \widetilde{\omega}_{\beta} f(\delta)_{L^{p}} \leq \widetilde{\omega}(\delta)\right\},
$$

where $\widetilde{\omega}$ is positive, with $\widetilde{\omega}(0)=0$, and almost nondecreasing continuous function.

Theorem 4. Let $f \in L^{p}(1 \leq p \leq \infty)$. If the entries of matrix $B$ satisfy condition (22) for $0 \leq \mu \leq \nu$ and if matrix $A$ is a maximal or minimal hump matrix with $k_{0}^{-1}=O\left(n^{-1}\right)$ such that $\left(a_{n, k}\right)_{k=0}^{n} \in$ MHBVS $\cup$ MRBVS, then

$$
\begin{aligned}
& \left\|\widetilde{T}_{n, A, B} f(\cdot)-\widetilde{f}\left(\cdot, \frac{\pi}{n+1}\right)\right\|_{L^{p}} \\
& \quad=O\left((n+1)^{\beta}\left[\frac{1}{n+1} \sum_{k=0}^{n} \widetilde{\omega}_{\beta} f\left(\frac{\pi}{k+1}\right)_{L^{p}}\right]\right),
\end{aligned}
$$

where $0 \leq \beta \leq 1$.

Theorem 5. Let $f \in L^{p}(\widetilde{\omega})_{\beta}$ with $1<p<\infty$, where $\widetilde{\omega}$ instead of $\widetilde{w}_{x}$ satisfies (24) with some $\beta \geq 0$. If the entries of matrix $B$ satisfy condition (22) for $0 \leq \mu \leq \nu$ and if matrix $A$ is 
a maximal or minimal hump matrix with $k_{0}^{-1}=O\left(n^{-1}\right)$ such that $\left(a_{n, k}\right)_{k=0}^{n} \in$ MHBVS $\cup$ MRBVS, then

$$
\begin{aligned}
& \left\|\widetilde{T}_{n, A, B} f(\cdot)-\widetilde{f}(\cdot)\right\|_{L^{p}} \\
& \quad=O\left((n+1)^{\beta}\left[\frac{1}{n+1} \sum_{k=0}^{n} \widetilde{\omega}\left(\frac{\pi}{k+1}\right)\right]\right) .
\end{aligned}
$$

Finally, we give corollary and remarks as an application of our results.

Taking $a_{n, r}=p_{n-r} / \sum_{\nu=0}^{n} p_{\nu}$ when $r=0,1,2, \ldots, n$ and $a_{n, r}=0$ when $r>n$ with $p_{\nu}>0, p_{\nu} \leq p_{\nu+1}$ and $b_{r, k}=$ $\left(\begin{array}{l}r \\ k\end{array}\right) \gamma^{k} /(1+\gamma)^{r}$ when $k=0,1,2, \ldots, r$ and $b_{n, r}=0$ when $k>r$ with $\gamma>0$, Theorem 2 (Theorem 3 analogously) implies the following.

Corollary 6. If $f \in L^{1}$, then

$$
\begin{aligned}
& \mid \frac{1}{\sum_{\nu=0}^{n} p_{\nu}} \sum_{r=0}^{n} \frac{p_{r}}{(1+\gamma)^{r}} \sum_{k=0}^{r}\left(\begin{array}{l}
r \\
k
\end{array}\right) \gamma^{k} \widetilde{S}_{k} f(x) \\
& -\tilde{f}\left(x, \frac{\pi}{n+1}\right) \mid \\
& \quad=O\left((n+1)^{\beta}\left[\frac{1}{n+1} \sum_{k=0}^{n} \widetilde{w}_{x}^{1} f\left(\frac{\pi}{k+1}\right)_{\beta}\right]\right), \\
& \frac{1}{\sum_{\nu=0}^{n} p_{\nu}} \sum_{r=0}^{n} \frac{p_{n-r}}{(1+\gamma)^{r}} \sum_{k=0}^{r}\left(\begin{array}{l}
r \\
k
\end{array}\right) \gamma^{k} \widetilde{S}_{k} f(x) \\
& -\widetilde{f}\left(x, \frac{\pi}{n+1}\right) \mid \\
& =O\left((n+1)^{\beta}\left[\frac{1}{n+1} \sum_{k=0}^{n} \widetilde{w}_{x}^{1} f\left(\frac{\pi}{k+1}\right)_{\beta}\right]\right),
\end{aligned}
$$

for almost all considered $x$, where $0 \leq \beta \leq 1, p_{\nu}>0, p_{\nu} \leq p_{\nu+1}$, and $\gamma>0$.

Remark 7. In special case, if $p_{r}=p_{n-r}=1$, then

$$
\begin{gathered}
\left|\frac{1}{n+1} \sum_{r=0}^{n} \frac{1}{(1+\gamma)^{r}} \sum_{k=0}^{r}\left(\begin{array}{l}
r \\
k
\end{array}\right) \gamma^{k} \widetilde{S}_{k} f(x)-\tilde{f}\left(x, \frac{\pi}{n+1}\right)\right| \\
=O\left((n+1)^{\beta}\left[\frac{1}{n+1} \sum_{k=0}^{n} \widetilde{w}_{x}^{1} f\left(\frac{\pi}{k+1}\right)_{\beta}\right]\right),
\end{gathered}
$$

and if $f \in \operatorname{Lip}(\alpha, p)$, then

$$
\begin{gathered}
\left|\frac{1}{n+1} \sum_{r=0}^{n} \frac{1}{(1+\gamma)^{r}} \sum_{k=0}^{r}\left(\begin{array}{l}
r \\
k
\end{array}\right) \gamma^{k} \widetilde{S}_{k} f(x)-\tilde{f}\left(x, \frac{\pi}{n+1}\right)\right| \\
\quad= \begin{cases}O\left((n+1)^{\beta-\alpha}\right) & \text { when } 0<\alpha<1 \\
O\left((n+1)^{\beta-1} \log (n+1)\right) & \text { when } \alpha=1,\end{cases}
\end{gathered}
$$

for almost all considered $x$, where $0 \leq \beta \leq 1$ and $\gamma>0$.
Remark 8. Taking $\beta=0$, we have, by Theorem 3 with $\widetilde{w}_{x}(\delta)=$ $\delta^{\alpha}$, for $0<\alpha<1$ and $\alpha p>1$, the estimate like in [12] with the better order of approximation without any additional assumptions.

Remark 9. Analyzing the proofs of Theorems 2-5, we can deduce that, taking the assumption $\left(a_{n, k}\right)_{k=0}^{n} \in$ RBVS or $\left(a_{n, k}\right)_{k=0}^{n} \in$ HBVS instead of $\left(a_{n, k}\right)_{k=0}^{n} \in$ MRBVS or $\left(a_{n, k}\right)_{k=0}^{n} \epsilon$ MHBVS, respectively, we obtain the results like that from [13].

\section{Auxiliary Results}

We begin this section by some notations following A. Zygmund [1, Section 5 of Chapter II].

It is clear that

$$
\begin{aligned}
\widetilde{S}_{k} f(x) & =-\frac{1}{\pi} \int_{-\pi}^{\pi} f(x+t) \widetilde{D}_{k}(t) d t \\
\widetilde{T}_{n, A, B} f(x) & =-\frac{1}{\pi} \int_{-\pi}^{\pi} f(x+t) \sum_{r=0}^{n} \sum_{k=0}^{r} a_{n, r} b_{r, k} \widetilde{D_{k}}(t) d t,
\end{aligned}
$$

where

$$
\widetilde{D_{k}}(t)=\sum_{v=0}^{k} \sin v t=\frac{\cos (t / 2)-\cos ((2 k+1) t / 2)}{2 \sin (t / 2)}
$$

Hence

$$
\begin{aligned}
\widetilde{T}_{n, A, B} f(x)-\widetilde{f}\left(x, \frac{\pi}{n+1}\right) \\
=-\frac{1}{\pi} \int_{0}^{\pi /(n+1)} \psi_{x}(t) \sum_{r=0}^{n} \sum_{k=0}^{r} a_{n, r} b_{r, k} \widetilde{D_{k}}(t) d t \\
\quad+\frac{1}{\pi} \int_{\pi /(n+1)}^{\pi} \psi_{x}(t) \sum_{r=0}^{n} \sum_{k=0}^{r} a_{n, r} b_{r, k} \widetilde{D_{k}^{\circ}}(t) d t, \\
\widetilde{T}_{n, A, B} f(x)-\tilde{f}(x) \\
=\frac{1}{\pi} \int_{0}^{\pi} \psi_{x}(t) \sum_{r=0}^{n} \sum_{k=0}^{r} a_{n, r} b_{r, k} \widetilde{D_{k}^{\circ}}(t) d t,
\end{aligned}
$$

where

$$
\widetilde{D_{k}^{\circ}}(t)=\frac{1}{2} \cot \frac{t}{2}-\sum_{\nu=0}^{k} \sin v t=\frac{\cos ((2 k+1) t / 2)}{2 \sin (t / 2)} .
$$

Now, we formulate some estimates for the conjugate Dirichlet kernels.

Lemma 10 (see [1]). If $0<|t| \leq \pi / 2$, then

$$
\left|\widetilde{D_{k}^{\circ}}(t)\right| \leq \frac{\pi}{2|t|}
$$

and for any real t one has

$$
\begin{aligned}
& \left|\widetilde{D_{k}}(t)\right| \leq \frac{1}{2} k(k+1)|t|, \\
& \left|\widetilde{D_{k}}(t)\right| \leq k+1 .
\end{aligned}
$$


Lemma 11. Let $\left(b_{r, k}\right)_{k=0}^{r}$ be such that (22) for $0 \leq \mu \leq \nu$ holds. we obtain If $\left(a_{n, k}\right)_{k=0}^{n} \in$ MRBVS, then

$$
\left|\sum_{r=0}^{n} \sum_{k=0}^{r} a_{n, r} b_{r, k} \widetilde{D_{k}^{\circ}}(t)\right| \ll \tau A_{n, \tau},
$$

and if $\left(a_{n, k}\right)_{k=0}^{n} \in$ MHBVS, then

$$
\left|\sum_{r=0}^{n} \sum_{k=0}^{r} a_{n, r} b_{r, k} \widetilde{D_{k}^{\circ}}(t)\right| \ll \tau \bar{A}_{n, n-\tau}
$$

with $\tau=[\pi / t]$ and $t \in[\pi /(n+1), \pi]$, for $n=0,1,2, \ldots$

Proof. Let

$$
K_{n}(t):=\sum_{r=0}^{n} \sum_{k=0}^{r} a_{n, r} b_{r, k} \cos \frac{(2 k+1) t}{2} .
$$

The relation $\left(a_{n, k}\right)_{k=0}^{n} \in$ MRBVS implies

$$
\begin{aligned}
a_{n, s}-a_{n, m} \leq & \left|a_{n, m}-a_{n, s}\right| \leq \sum_{k=m}^{s-1}\left|a_{n, k}-a_{n, k+1}\right| \\
\ll \sum_{k=r}^{n-1}\left|a_{n, k}-a_{n, k+1}\right| \ll & \frac{1}{r+1} \sum_{k \geq r / 2}^{r} a_{n, k}, \\
& \quad(0 \leq r \leq m<s \leq n)
\end{aligned}
$$

whence

$$
a_{n, s} \ll a_{n, m}+\frac{1}{r+1} \sum_{k \geq r / 2}^{r} a_{n, k}, \quad(0 \leq r \leq m<s \leq n),
$$

and thus, by our assumption,

$$
\left|\sum_{l=\tau+1}^{r} \sum_{k=0}^{l} b_{l, k} \cos \frac{(2 k+1) t}{2}\right| \ll \tau,
$$

$$
\begin{aligned}
& \left|K_{n}(t)\right|=\left|\left(\sum_{r=0}^{\tau}+\sum_{r=\tau+1}^{n}\right) a_{n, r} \sum_{k=0}^{r} b_{r, k} \cos \frac{(2 k+1) t}{2}\right| \\
& \leq \sum_{r=0}^{\tau} a_{n, r} \sum_{k=0}^{r} b_{r, k}+\sum_{r=\tau+1}^{n-1}\left|a_{n, r}-a_{n, r+1}\right| \\
& \quad \cdot\left|\sum_{l=\tau+1}^{r} \sum_{k=0}^{l} b_{l, k} \cos \frac{(2 k+1) t}{2}\right| \\
& \quad+a_{n, n}\left|\sum_{l=\tau+1}^{n} \sum_{k=0}^{l} b_{l, k} \cos \frac{(2 k+1) t}{2}\right| \ll A_{n, \tau} \\
& \quad+\left(\frac{1}{\tau+1} \sum_{k \geq \tau / 2}^{\tau} a_{n, k}+\frac{1}{\tau+1} \sum_{k \geq \tau / 2}^{\tau} a_{n, n}\right) \tau \leq A_{n, \tau}
\end{aligned}
$$

$$
\begin{aligned}
& +\left[\frac{1}{\tau+1} \sum_{k \geq \tau / 2}^{\tau} a_{n, k}\right. \\
& \left.+\frac{1}{\tau+1} \sum_{k \geq \tau / 2}^{\tau}\left(a_{n, k}+\frac{1}{k+1} \sum_{l \geq k / 2}^{k} a_{n, l}\right)\right] \tau \leq A_{n, \tau}
\end{aligned}
$$$$
+\left[\frac{1}{\tau+1} \sum_{k \geq \tau / 2}^{\tau} a_{n, k}\right.
$$

$$
\begin{aligned}
& \left.+\frac{1}{\tau+1} \sum_{k \geq \tau / 2}^{\tau}\left(a_{n, k}+\frac{1}{\tau / 2+1} \sum_{l \geq \tau / 4}^{\tau} a_{n, l}\right)\right] \tau \leq A_{n, \tau} \\
& +\left[\frac{1}{\tau+1} \sum_{k \geq \tau / 2}^{\tau} a_{n, k}\right. \\
& \left.+\frac{1}{\tau+1} \sum_{k \geq \tau / 2}^{\tau}\left(a_{n, k}+\frac{1}{\tau / 2+1} \sum_{l \geq \tau / 4}^{\tau} a_{n, l}\right)\right] \tau \leq A_{n, \tau} \\
& +\left[\frac{1}{\tau+1} \sum_{k \geq \tau / 2}^{\tau} a_{n, k}+\frac{1}{\tau+1} \sum_{k \geq \tau / 2}^{\tau} a_{n, k}+\frac{1}{\tau+1} \sum_{l=0}^{\tau} a_{n, l}\right]
\end{aligned}
$$

$$
\cdot \tau \leq A_{n, \tau}+3 \sum_{l=0}^{\tau} a_{n, l}=4 A_{n, \tau} \cdot
$$

The relation $\left(a_{n, k}\right)_{k=0}^{n} \in$ MHBVS implies

$$
\begin{array}{r}
a_{n, m}-a_{n, s} \leq\left|a_{n, m}-a_{n, s}\right| \leq \sum_{k=m}^{s-1}\left|a_{n, k}-a_{n, k+1}\right| \\
\ll \sum_{k=0}^{r-1}\left|a_{n, k}-a_{n, k+1}\right| \ll \frac{1}{n-r+1} \sum_{k=r}^{n} a_{n, k}, \\
\quad(0 \leq m<s \leq r \leq n)
\end{array}
$$


whence

$$
a_{n, m} \ll a_{n, s}+\frac{1}{n-r+1} \sum_{k=r}^{n} a_{n, k},
$$

$(0 \leq m<s \leq r \leq n)$,

and thus, by our assumption,

$$
\left|\sum_{l=0}^{r} \sum_{k=0}^{l} b_{l, k} \cos \frac{(2 k+1) t}{2}\right| \ll \tau,
$$

we get

$$
\begin{aligned}
& \left|K_{n}(t)\right|=\left|\left(\sum_{r=n-\tau}^{n}+\sum_{r=0}^{n-\tau}\right) a_{n, r} \sum_{k=0}^{r} b_{r, k} \cos \frac{(2 k+1) t}{2}\right| \\
& =\bar{A}_{n, n-\tau}+\sum_{r=0}^{n-\tau-1}\left|a_{n, r}-a_{n, r+1}\right| \\
& \left|\sum_{l=0}^{r} \sum_{k=0}^{l} b_{l, k} \cos \frac{(2 k+1) t}{2}\right| \\
& +a_{n, n-\tau-1}\left|\sum_{l=0}^{n-\tau} \sum_{k=0}^{l} b_{l, k} \cos \frac{(2 k+1) t}{2}\right| \ll \bar{A}_{n, n-\tau} \\
& +\left[\frac{1}{\tau+1} \sum_{k=n-\tau}^{n} a_{n, k}+a_{n, n-\tau-1}\right] \tau \ll \bar{A}_{n, n-\tau} \\
& +\left[\frac{1}{\tau+1} \sum_{k=n-\tau}^{n} a_{n, k}+\frac{1}{\tau+1} \sum_{k=n-\tau}^{n-\tau / 2} a_{n, n-\tau-1}\right] \tau \\
& \ll \bar{A}_{n, n-\tau}+\left[\frac{1}{\tau+1} \sum_{k=n-\tau}^{n} a_{n, k}\right. \\
& \left.+\frac{1}{\tau+1} \sum_{k=n-\tau}^{n-\tau / 2}\left(a_{n, k}+\frac{1}{\tau+1} \sum_{\nu=n-\tau / 2}^{n} a_{n, \nu}\right)\right] \tau \\
& \leq \bar{A}_{n, n-\tau}+\sum_{k=n-\tau}^{n} a_{n, k}+\sum_{k=n-\tau}^{n} \frac{1}{\tau+1} \sum_{\nu=n-\tau}^{n} a_{n, \nu} \\
& \ll \bar{A}_{n, n-\tau} .
\end{aligned}
$$

Now, our proof is complete.

Lemma 12. If matrix $A$ is a maximal or minimal hump matrix with $k_{0}^{-1}=O\left(n^{-1}\right)$, then

$$
\frac{n+1}{m+1} \sum_{r=0}^{m} a_{n, r}=O(1)
$$

or

$$
\frac{n+1}{m+1} \sum_{r=0}^{m} a_{n, n-r}=O(1)
$$

for $m=0,1,2, \ldots$, respectively.
Proof. Since the above formulas are similar, we prove the first one only. If $0<m \leq k_{0}$, then

$$
\begin{aligned}
& \frac{1}{m} \sum_{r=0}^{m-1} a_{n, r}-\frac{1}{m+1} \sum_{r=0}^{m} a_{n, r} \\
& \quad=\frac{1}{m(m+1)} \sum_{r=0}^{m} a_{n, r}-\frac{a_{n, m}}{m} \\
& \quad=\frac{1}{m(m+1)} \sum_{r=0}^{m}\left(a_{n, r}-a_{n, m}\right) \leq 0,
\end{aligned}
$$

whence $(1 /(m+1)) \sum_{r=0}^{m} a_{n, r}$ nondecreases with respect to $m$ and therefore

$$
\begin{aligned}
\frac{1}{m+1} \sum_{r=0}^{m} a_{n, r} & \leq \frac{1}{k_{0}+1} \sum_{r=0}^{k_{0}} a_{n, r} \leq \frac{1}{k_{0}+1} \sum_{r=0}^{n} a_{n, r} \\
& =\frac{1}{k_{0}+1}=O\left(n^{-1}\right) .
\end{aligned}
$$

But if $m \geq k_{0}$, then

$$
\begin{aligned}
\frac{1}{m+1} \sum_{r=0}^{m} a_{n, r} & \leq \frac{1}{k_{0}+1} \sum_{r=0}^{m} a_{n, r} \leq \frac{1}{k_{0}+1} \sum_{r=0}^{n} a_{n, r} \\
& =\frac{1}{k_{0}+1}=O\left(n^{-1}\right)
\end{aligned}
$$

and our proof is complete.

Lemma 13. If $f \in L^{p}(1 \leq p \leq \infty)$ and $\beta \geq 0$, then

$$
\widetilde{w}_{x}^{p} f\left(\frac{\pi}{n+1}\right)_{\beta} \leq\left\{\frac{2}{n+1} \sum_{r=0}^{n}\left[\widetilde{w}_{x}^{p} f\left(\frac{\pi}{r+1}\right)_{\beta}\right]^{p}\right\}^{1 / p}
$$

holds for every natural $n$ and all real $x$.

Proof. The proof follows by the easy account

$$
\begin{aligned}
& {\left[\widetilde{w}_{x}^{p} f\left(\frac{\pi}{n+1}\right)_{\beta}\right]^{p}} \\
& \quad=\frac{n+1}{\pi} \int_{0}^{\pi /(n+1)}\left|\psi_{x}(u)\right|^{p} d u \sum_{r=0}^{n} \frac{2(r+1)}{(n+1)(n+2)} \\
& \leq \frac{2}{n+1} \sum_{r=0}^{n} \frac{(r+1)(n+1)}{\pi(n+2)} \int_{0}^{\pi /(n+1)}\left|\psi_{x}(u)\right|^{p} d u \\
& \leq \frac{2}{n+1} \sum_{r=0}^{n} \frac{r+1}{\pi} \int_{0}^{\pi /(r+1)}\left|\psi_{x}(u)\right|^{p} d u \\
& =2 \frac{1}{n+1} \sum_{r=0}^{n}\left[\widetilde{w}_{x}^{p} f\left(\frac{\pi}{r+1}\right)_{\beta}\right]^{p} .
\end{aligned}
$$

Now, our proof is complete. 


\section{Proofs of the Results}

4.1. Proof of Theorem 2. We start with the obvious relations

$$
\begin{aligned}
& \widetilde{T}_{n, A, B} f(x)-\tilde{f}\left(x, \frac{\pi}{n+1}\right) \\
& =-\frac{1}{\pi} \int_{0}^{\pi /(n+1)} \psi_{x}(t) \sum_{r=0}^{n} \sum_{k=0}^{r} a_{n, r} b_{r, k} \widetilde{D_{k}}(t) d t \\
& \quad+\frac{1}{\pi} \int_{\pi /(n+1)}^{\pi} \psi_{x}(t) \sum_{r=0}^{n} \sum_{k=0}^{r} a_{n, r} b_{r, k} \widetilde{D_{k}^{o}}(t)=\widetilde{I_{1}}+\widetilde{I_{2}^{o}}, \\
& \left|\widetilde{T}_{n, A} f(x)-\widetilde{f}\left(x, \frac{\pi}{n+1}\right)\right| \leq\left|\widetilde{I_{1}}\right|+\left|\widetilde{I_{2}^{o}}\right| .
\end{aligned}
$$

By Lemmas 10 and 13, we have

$$
\begin{aligned}
\left|\widetilde{I}_{1}\right| & \ll(n+1)^{2} \int_{0}^{\pi /(n+1)} t\left|\psi_{x}(t)\right| d t \\
& \leq(n+1)^{2} \int_{0}^{\pi /(n+1)} t\left|\psi_{x}(t)\right| \sin ^{\beta} \frac{t}{2} \sin ^{-\beta} \frac{t}{2} d t \\
& \ll(n+1)^{2} \int_{0}^{\pi /(n+1)}\left|\psi_{x}(t)\right| \sin ^{\beta} \frac{t}{2} d t\left(\frac{\pi}{n+1}\right)^{1-\beta} \\
& \ll(n+1)^{\beta} \widetilde{w}_{x}^{1} f\left(\frac{\pi}{n+1}\right)_{\beta} \\
& \ll(n+1)^{\beta-1} \sum_{k=0}^{n} \widetilde{w}_{x}^{1} f\left(\frac{\pi}{k+1}\right)_{\beta},
\end{aligned}
$$

for $\beta \in[0,1]$.

Using Lemmas 11 and 12 we obtain

$$
\begin{aligned}
\left|\widetilde{I_{2}^{\circ}}\right| & \ll \int_{\pi /(n+1)}^{\pi} \frac{\left|\psi_{x}(t)\right|}{t} \sum_{k=0}^{\tau} a_{n, k} d t \\
& \ll \int_{\pi /(n+1)}^{\pi} \frac{\left|\psi_{x}(t)\right|}{t} \frac{\tau}{n+1} d t
\end{aligned}
$$

or

$$
\begin{aligned}
\left|\widetilde{I_{2}^{\circ}}\right| & \ll \int_{\pi /(n+1)}^{\pi} \frac{\left|\psi_{x}(t)\right|}{t} \sum_{k=n-\tau}^{n} a_{n, k} d t \\
& =\int_{\pi /(n+1)}^{\pi} \frac{\left|\psi_{x}(t)\right|}{t} \sum_{k=0}^{\tau} a_{n, n-k} d t \\
& \ll \int_{\pi /(n+1)}^{\pi} \frac{\left|\psi_{x}(t)\right|}{t} \frac{\tau}{n+1} d t,
\end{aligned}
$$

whence

$$
\begin{aligned}
\left|\widetilde{I}_{2}^{\circ}\right| & \ll \frac{1}{n+1} \int_{\pi /(n+1)}^{\pi} t^{-2}\left[\left|\psi_{x}(t)\right| \sin ^{\beta} \frac{t}{2}\right] \frac{1}{\sin ^{\beta}(t / 2)} d t \\
& \ll(n+1)^{\beta} \frac{1}{n+1} \int_{\pi /(n+1)}^{\pi} t^{-2}\left[\left|\psi_{x}(t)\right| \sin ^{\beta} \frac{t}{2}\right] d t \\
& =(n+1)^{\beta} \frac{1}{n+1} \\
& \cdot \int_{\pi /(n+1)}^{\pi} t^{-2} \frac{d}{d t}\left[\int_{0}^{t}\left|\psi_{x}(u)\right| \sin ^{\beta} \frac{u}{2} d u\right] d t=(n \\
& +1)^{\beta-1}\left\{\left[t^{-2} \int_{0}^{t}\left|\psi_{x}(u)\right| \sin ^{\beta} \frac{u}{2} d u\right]_{t=\pi /(n+1)}^{\pi}\right. \\
& \left.+2 \int_{\pi /(n+1)}^{\pi} t^{-3}\left[\int_{0}^{t}\left|\psi_{x}(u)\right| \sin ^{\beta} \frac{u}{2} d u\right] d t\right\}=(n \\
& +1)^{\beta-1} \\
& \cdot\left\{\left[\pi^{-1} \widetilde{w}_{x}^{1} f(\pi)_{\beta}-\left(\frac{\pi}{n+1}\right)^{-1} \widetilde{w}_{x}^{1} f\left(\frac{\pi}{n+1}\right)_{\beta}\right]\right. \\
& \left.+2 \int_{\pi /(n+1)}^{\pi} t^{-2} \widetilde{w}_{x}^{1} f(t)_{\beta} d t\right\} \ll(n+1)^{\beta-1} \\
& \cdot\left\{\sum_{k=0}^{n} \widetilde{w}_{x}^{1} f\left(\frac{\pi}{k+1}\right)_{\beta}\right. \\
& \left.+\int_{1}^{n+1} \widetilde{w}_{x}^{1} f\left(\frac{\pi}{t}\right)_{\beta} d t\right\} \ll(n+1)^{\beta-1} \\
& \cdot \sum_{k=0}^{n} \widetilde{w}_{x}^{1} f\left(\frac{\pi}{k+1}\right)_{\beta} \cdot
\end{aligned}
$$

Collecting these estimates, we obtain the desired result.

4.2. Proof of Theorem 3. We start with the obvious relations

$$
\begin{aligned}
& \widetilde{T}_{n, A} f(x)-\tilde{f}(x) \\
& =\frac{1}{\pi} \int_{0}^{\pi /(n+1)} \psi_{x}(t) \sum_{r=0}^{n} \sum_{k=0}^{r} a_{n, r} b_{r, k} \widetilde{D_{k}^{\circ}}(t) d t \\
& \quad+\frac{1}{\pi} \int_{\pi /(n+1)}^{\pi} \psi_{x}(t) \sum_{r=0}^{n} \sum_{k=0}^{r} a_{n, r} b_{r, k} \widetilde{D_{k}^{\circ}}(t) d t \\
& =\widetilde{I_{1}^{\circ}}+\widetilde{I_{2}^{\circ}}, \\
& \left|\widetilde{T}_{n, A} f(x)-\widetilde{f}(x)\right| \leq\left|\widetilde{I_{1}^{\circ}}\right|+\left|\widetilde{I_{2}^{\circ}}\right| .
\end{aligned}
$$


By the Hölder inequality $(1 / p+1 / q=1)$, Lemma 10, (25), and (24),

$$
\begin{aligned}
\left|\widetilde{I}_{1}^{o}\right| & \ll \int_{0}^{\pi /(n+1)} \frac{\left|\psi_{x}(t)\right|}{t} d t \\
& \leq\left\{\int_{0}^{\pi /(n+1)}\left[\frac{\left|\psi_{x}(t)\right|}{\widetilde{w}_{x}(t)} \sin ^{\beta} \frac{t}{2}\right]^{p} d t\right\}^{1 / p} \\
& \cdot\left\{\int_{0}^{\pi /(n+1)}\left[\frac{\widetilde{w}_{x}(t)}{t \sin ^{\beta}(t / 2)}\right]^{q} d t\right\}^{1 / q} \ll(n+1)^{\beta} \\
\cdot & \widetilde{w}_{x}\left(\frac{\pi}{n+1}\right)=(n+1)^{\beta-1} \widetilde{w}_{x}\left(\frac{\pi}{n+1}\right) \sum_{k=0}^{n} 1 \\
& \ll(n+1)^{\beta-1} \sum_{k=0}^{n} \widetilde{w}_{x}\left(\frac{\pi}{k+1}\right) .
\end{aligned}
$$

We can estimate the term $\left|\widetilde{I_{2}^{\circ}}\right|$ by the same way like in the proof of Theorem 2:

$$
\begin{aligned}
\left|\widetilde{I}_{2}^{\circ}\right| & \ll \int_{\pi /(n+1)}^{\pi} \frac{\left|\psi_{x}(t)\right|}{t} \sum_{k=n-\tau}^{n} a_{n, k} d t \\
& =\int_{\pi /(n+1)}^{\pi} \frac{\left|\psi_{x}(t)\right|}{t} \sum_{k=0}^{\tau} a_{n, n-k} d t \\
& \ll \int_{\pi /(n+1)}^{\pi} \frac{\left|\psi_{x}(t)\right|}{t} \frac{\tau}{n+1} d t \\
& \ll(n+1)^{\beta-1} \sum_{k=0}^{n} \widetilde{w}_{x}^{1} f\left(\frac{\pi}{k+1}\right)_{\beta} \\
& \ll(n+1)^{\beta-1} \sum_{k=0}^{n} \widetilde{w}_{x}\left(\frac{\pi}{k+1}\right) .
\end{aligned}
$$

Collecting these estimates, we obtain the desired result.

4.3. Proofs of Theorems 4-5. The proofs are similar to these above and follow from the evident inequality

$$
\left\|\widetilde{w}^{p} f(\delta)_{\beta}\right\|_{L^{p}} \ll \widetilde{\omega}_{\beta} f(\delta)_{L^{p}}
$$

and additionally in case of Theorem 5 from the estimate

$$
\begin{aligned}
& \left\|\left\{\int_{0}^{\pi /(n+1)}\left(\frac{|\psi \cdot(t)|}{\widetilde{\omega}(t)}\right)^{p} \sin ^{\beta p} \frac{t}{2} d t\right\}^{1 / p}\right\|_{L^{p}} \\
& \leq\left\{\int_{0}^{\pi /(n+1)}\left(\frac{\left\|\psi \cdot \sin ^{\beta} \frac{t}{2}\right\|_{L^{p}}}{\widetilde{\omega}(t)}\right) d t\right\} \\
& \quad \leq\left\{\int_{0}^{\pi /(n+1)}\left(\frac{\widetilde{\omega}_{\beta} f(t)_{L^{p}}}{\widetilde{\omega}(t)}\right)^{p} d t\right\}^{1 / p} \\
& \quad \leq\left\{\int_{0}^{\pi /(n+1)} d t\right\}^{1 / p}=O_{x}\left((n+1)^{-1 / p}\right)
\end{aligned}
$$

for $f \in L^{p}(\widetilde{\omega})_{\beta}$.
4.4. Proof of Corollary 6. First of all we note that our matrix $A$ is the hump matrix with $k_{0}=n$.

Next, we have to verify the assumptions of Lemma 11. For the first one, we note that any nondecreasing sequence belongs to the class MRBVS and any nonincreasing sequence belongs to the class MHBVS. The second one follows from the following calculations:

$$
\begin{aligned}
\left|\sum_{r=\mu}^{\nu}(1+\gamma)^{-r} \sum_{k=0}^{r}\left(\begin{array}{l}
r \\
k
\end{array}\right) \gamma^{k} \cos \frac{(2 k+1) t}{2}\right| \\
=\left|\operatorname{Re} \sum_{r=\mu}^{\nu}(1+\gamma)^{-r} \sum_{k=0}^{r}\left(\begin{array}{l}
r \\
k
\end{array}\right) \gamma^{k} \exp \frac{(2 k+1) i t}{2}\right| \\
=\left|\operatorname{Re} \sum_{r=\mu}^{\nu}(1+\gamma)^{-r} \exp \frac{i t}{2} \sum_{k=0}^{r}\left(\begin{array}{l}
r \\
k
\end{array}\right) \gamma^{k} \exp i k t\right| \\
=\left|\operatorname{Re} \sum_{r=\mu}^{\nu}(1+\gamma)^{-r} \exp \frac{i t}{2}(1+\gamma \exp i t)^{r}\right| \\
=\left|\operatorname{Re}\left[\exp \frac{i t}{2} \sum_{r=\mu}^{\nu}\left(\frac{1+\gamma \exp i t}{1+\gamma}\right)^{r}\right]\right|=\mid \operatorname{Re}\left[\exp \frac{i t}{2}\right. \\
.\left(\frac{1+\gamma \exp i t}{1+\gamma}\right)^{\mu} \\
\left.. \frac{1-((1+\gamma \exp i t) /(1+\gamma))^{\nu-\mu+1}}{1-(1+\gamma \exp i t) /(1+\gamma)}\right] \mid \\
\quad \leq\left|\frac{1-((1+\gamma \exp i t) /(1+\gamma))^{\nu-\mu+1}}{1-(1+\gamma \exp i t) /(1+\gamma)}\right| \\
=\frac{2(1+\gamma)}{\gamma \sqrt{2} \sqrt{2 \sin { }^{2}(t / 2)}=\frac{1+\gamma}{\gamma|\sin (t / 2)|} \leq \frac{1+\gamma}{\gamma} \frac{\pi}{t}} \\
\quad \leq \frac{2(1+\gamma)}{|1-(1+\gamma \exp i t) /(1+\gamma)|} \\
=\frac{2(1+\gamma)}{|1+\gamma-1-\gamma \exp i t|} \frac{2(1+\gamma)}{\gamma|1-\exp i t|}
\end{aligned}
$$

Thus proof is complete.

\section{Conflict of Interests}

The authors declare that there is no conflict of interests regarding the publication of this paper. 


\section{References}

[1] A. Zygmund, Trigonometric Series, Cambridge, UK, 2002.

[2] L. Leindler, "On the degree of approximation of continuous functions," Acta Mathematica Hungarica, vol. 104, no. 1-2, pp. 105-113, 2004.

[3] L. Leindler, "Integrability conditions pertaining to Orlicz space," Journal of Inequalities in Pure and Applied Mathematics, vol. 8, no. 2, article 38, 6 pages, 2007.

[4] W. Lenski and B. Szal, "Approximation of functions from $L^{p}(\widetilde{w})_{\beta}$ by linear operators of conjugate Fourier series," Banach Center Publications, vol. 9, pp. 237-247, 2011.

[5] B. Szal, "A note on the uniform convergence and boundedness a generalized class of sine series," Commentationes Mathematicae, vol. 48, no. 1, pp. 85-94, 2008.

[6] V. N. Mishra and L. N. Mishra, "Trigonometric approximation of signals (functions) in $L^{p}$-norm," International Journal of Contemporary Mathematical Sciences, vol. 7, no. 19, pp. 909-918, 2012.

[7] M. L. Mittal, B. E. Rhoades, V. N. Mishra, and U. Singh, "Using infinite matrices to approximate functions of class $\operatorname{Lip}(\alpha$, p) using trigonometric polynomials," Journal of Mathematical Analysis and Applications, vol. 326, no. 1, pp. 667-676, 2007.

[8] K. Qureshi, "On the degree of approximation of functions belonging to the Lipschitz class by means of a conjugate series," Indian Journal of Pure and Applied Mathematics, vol. 12, no. 9, pp. 1120-1123, 1981.

[9] S. Lal and H. K. Nigam, "Degree of approximation of conjugate of a function belonging to $(\xi(t), p)$ class by matrix summability means of conjugate Fourier series," International Journal of Mathematics and Mathematical Sciences, vol. 27, no. 9, pp. 555$563,2001$.

[10] K. Qureshi, "On the degree of approximation of functions belonging to the class Lip $(\alpha, \mathrm{p})$ by means of a conjugate series," Indian Journal of Pure and Applied Mathematics, vol. 13, no. 5, pp. 560-563, 1982.

[11] S. Lal, "On the degree of approximation of conjugate of a function belonging to weighted $W\left(L^{p}, \xi(t)\right)$ class by matrixsummability means of conjugate series of a Fourier series," Tamkang Journal of Mathematics, vol. 31, no. 4, pp. 279-288, 2000.

[12] S. Sonker and U. Singh, "Degree of approximation of the conjugate of signals (functions) belonging to Lip $(\alpha, r)$-class by $(C, 1)(E, q)$ means of conjugate trigonometric Fourier series," Journal of Inequalities and Applications, vol. 2012, article 278, 2012.

[13] W. Lenski and B. Szal, "Approximation of functions belonging to the class $L^{\mathrm{p}}(\omega)_{\beta}$ by linear operators," Acta et Commentationes Universitatis Tartuensis de Mathematica, vol. 13, pp. 11-24, 2009. 


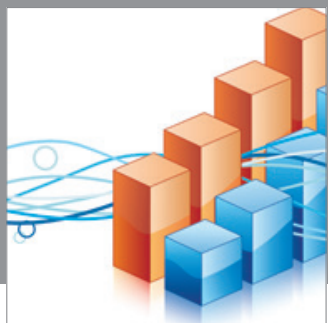

Advances in

Operations Research

mansans

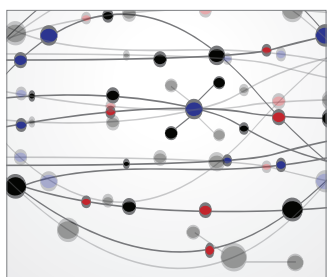

The Scientific World Journal
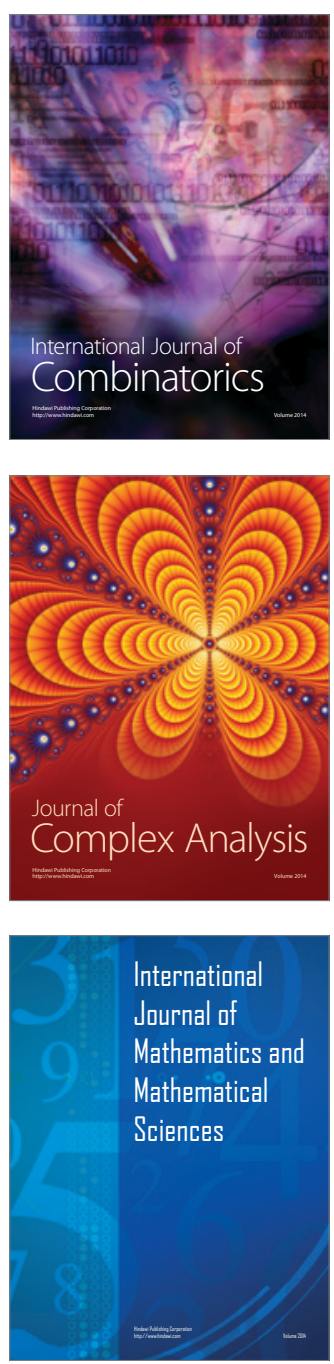
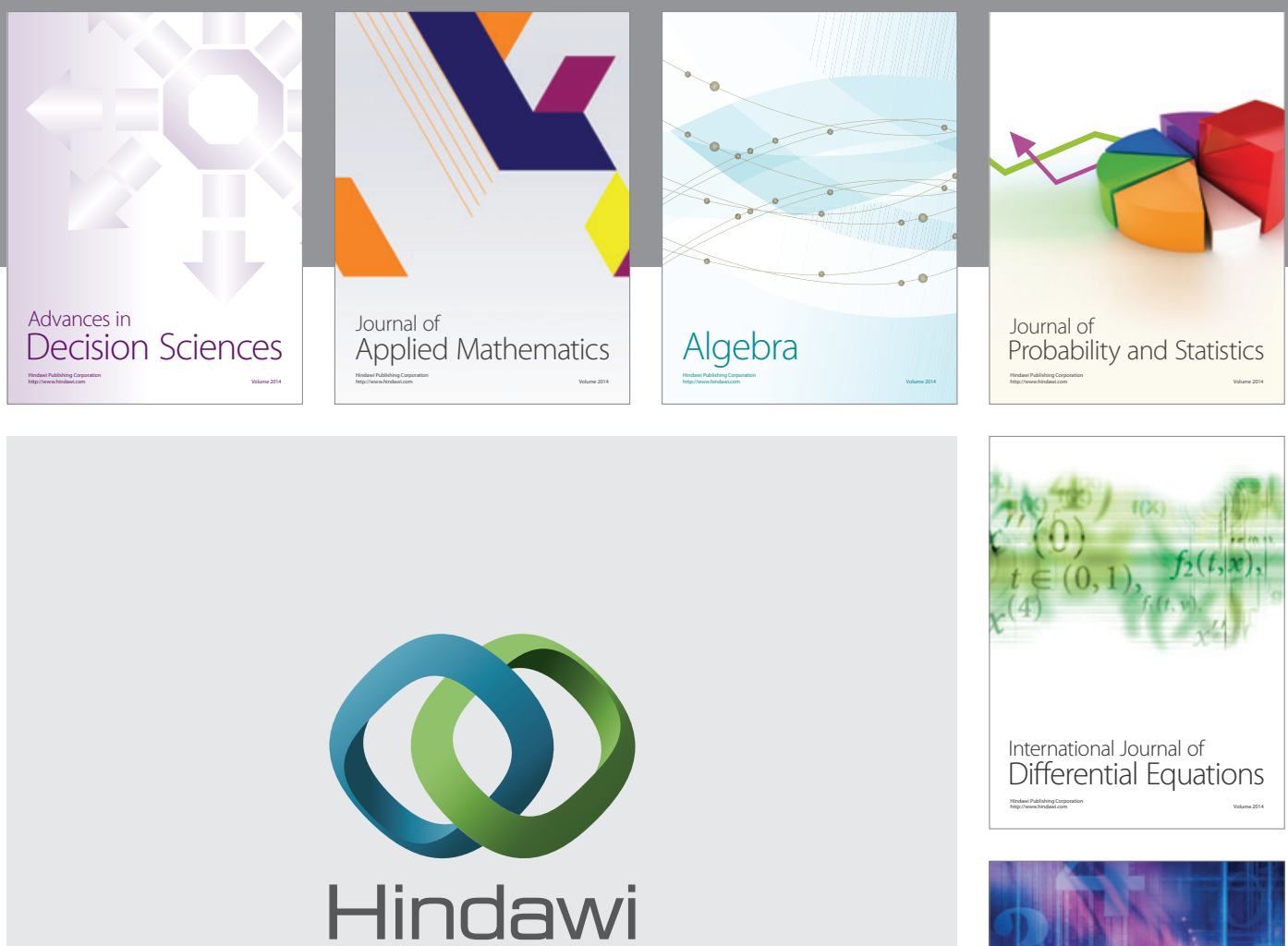

Submit your manuscripts at http://www.hindawi.com
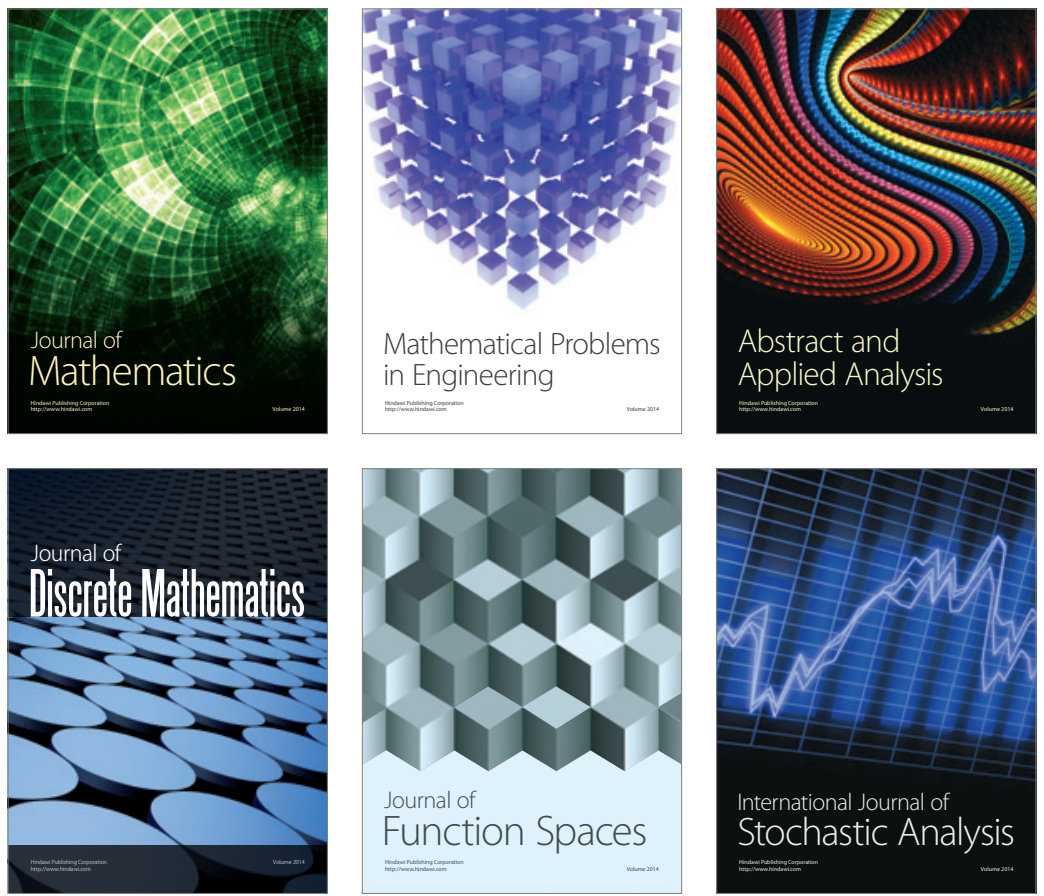

Journal of

Function Spaces

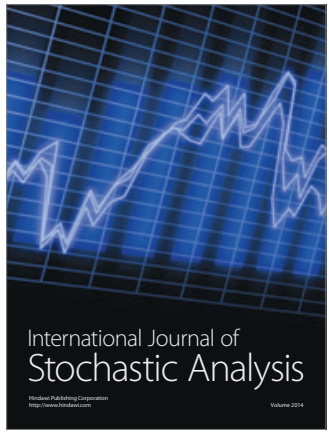

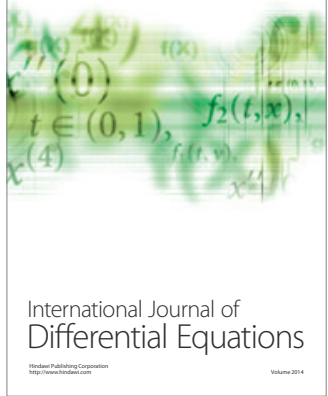
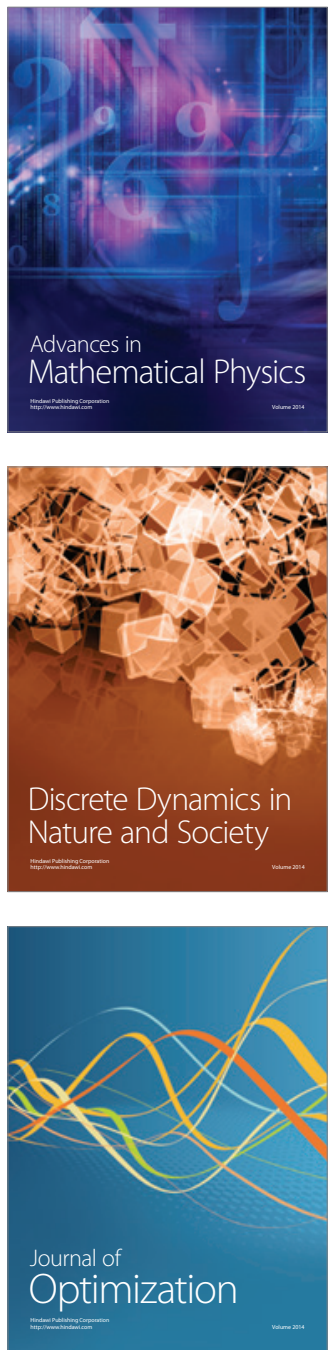